\title{
BISPECTRAL TECHNIQUES FOR SPHERICAL FUNCTIONS
}

\author{
Ramakrishna Kakarala*, Bruce M. Bennett**, Geoffrey J. Iverson**, and Michael D'Zmura** \\ *Dept. of Elect. \& Electronic Eng., Univ. of Auckland, Private Bag, Auckland, New Zealand \\ **Depts. of Math. and Cognitive Sci., Univ. of California, Irvine CA 92717, USA
}

\begin{abstract}
Spherical functions arise in geophysics, medical imaging, and computer graphics. This paper addresses two problems involving spherical functions: determining when two spherical functions are 3-D rotated copies of each other, and averaging several noisy observations of a rotating spherical function. Our solution to both problems uses the spherical bispectrum, which is the generalization of the wellknown Euclidean bispectrum. In this paper, we formulate the spherical bispectrum and show that it is invariant under 3-D rotation of the underlying function and unbiased in the presense of additive Gaussian noise. We demonstrate an algorithm for recovering spherical functions from their bispectra.
\end{abstract}

\section{INTRODUCTION}

Spherical functions (functions whose domain is a sphere) are useful for modelling the earth's gravitational field [1], describing the deformation of the heart surface during a cardiac cycle [2], and modelling reflectance from a spatially extended light source [3]. There are two general problems involving spherical functions that we address in this paper: how to determine when two given spherical functions are simply three-dimensional (3-D) rotated copies of each other; and how to average several noisy observations of a single spherical function that is undergoing an unknown 3$D$ rotation in between successive observations. For similar problems, when the functions involved are defined on the real line, bispectral techniques are appropriate for the following reasons [4]: (1) the bispectrum of any deterministic function on the real line is invariant under translation of the function; (2) the expected bispectrum of any zero-mean Gaussian noise process is identically zero; (3) the bispectrum of most deterministic functions on the real line contains enough information to enable recovery of the underlying function. In this paper we formulate the appropriate bispectrum for spherical functions, show that the new spherical bispectrum possesses attractive properties similar to (1)-(3) above, and demonstrate bispectral applications to the matching and averaging tasks mentioned above.

This work was supported by NSF Grant DIR9014278 to the Institute for Mathematical Behavioral Sciences, University of California, Irvine, R. D. Luce, Director. "E-mail address: R.KAKARALA@CCU1.AUKUNI.AC.NZ

\section{DEFINITIONS}

The bispectrum of any function $f$ on $\mathbb{R}^{n}$ is the Fourier transform of that function's triple correlation. In this section, we demonstrate how to obtain the spherical triple correlation from its well-known analogue on Euclidean space.

\section{A. Euclidean triple correlation}

Let $f$ be any complex-valued function in $L_{1}\left(\mathbb{R}^{n}\right)$. Its triple correlation $a_{3, f}$ is defined by the integral

$$
a_{3, f}(s, t)=\int_{\mathbf{R}^{n}} f(x)^{*} f(x+s) f(x+t) d x,
$$

where the asterisk * denotes complex-conjugation. Clearly, the triple correlation is invariant under translation of $f$, i.e., if there exists $t$ such that $h(x)=f(x+t)$ for all $x$, then $a_{3, h}=a_{3, f}$. The bispectrum $A_{3, f}$ is the Fourier transform of the triple correlation. One finds easily that

$$
A_{3, f}(u, v)=F(u) F(v) F(u+v)^{*},
$$

where $F$ is the Fourier transform of $f$ [4]. In many cases, the bispectrum $A_{3, f}$ is unique to the underlying function $f$ and its translates, i.e., if for some function $h$ we have that $A_{3, h}=A_{3, f}$, then there exists $t$ such that $h(x)=f(x+t)$ for all $x$. For example, this is true if $f$ is nonnegative and has compact support; for a detailed review of other cases where uniqueness holds, see [5].

The triple correlation for deterministic functions, as calculated in eq.(1), is unbiased in additive Gaussian noise. To make that precise, suppose that we observe over a compact region $K$ of $\mathbb{R}^{n}$ a signal $r$ of the form $r(x)=f(x)+n(x)$, where $f$ is a deterministic function and $n$ is a sample from a zero-mean stationary Gaussian process. If we subtract from $r$ its mean value $\bar{r}$ over $K$, and set $r^{\prime}=r-\bar{r}$, then we find that

$$
E\left[a_{3, r^{\prime}}(s, t)\right]=a_{3, f^{\prime}}(s, t) .
$$

Here $f^{\prime}$ denotes the zero-mean function $f-\bar{f}$.

\section{B. Spherical triple correlation}

The Euclidean triple correlation in eq.(1) is the integral of the Euclidean function $f$ multiplied by two independently translated copies of itself. Therefore, the appropriate spherical triple correlation should integrate the spherical function $f$ multiplied by two independently rotated copies of itself. To make the analogy even more direct, we define a new function $\tilde{f}$ on the $3-\mathrm{D}$ rotation group $\mathrm{SO}(3)$, in such a way that if $f$ undergoes a 3-D rotation, then $\tilde{f}$ undergoes a corresponding left translation on $\mathrm{SO}(3)$. We show later how

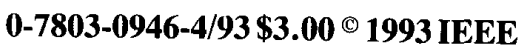


to construct $\tilde{f}$ from $f$, but for now let $\tilde{f}$ be an arbitrary function on $\mathrm{SO}(3)$. We define the triple correlation of $f$ to be the integral

$$
a_{3, \tilde{f}}(S, T)=\int_{S O(3)} \tilde{f}(R)^{*} \tilde{f}(R S) \tilde{f}(R T) d R .
$$

Here $\int d R$ is the unique normalized Haar integral on $\mathrm{SO}(3)$, which is invariant under either left or right translation of $\tilde{f}$, i.e.,

$$
\int \tilde{f}\left(R R_{0}\right) d R=\int \tilde{f}(R) d R=\int \tilde{f}\left(R_{0} R\right) d R .
$$

(The explicit form of the Haar integral in terms of Euler angles is contained in standard texts [6].) By applying (4) to eq. (3), it is easily seen that $a_{3, j}$ is invariant under left translation of $\dot{f}$ on $\mathrm{SO}(3)$.

We now apply eq.(3) to spherical functions. Let $S^{2}$ denote the sphere of unit radius in $\mathbb{R}^{3}$, and let $\hat{k}=[0,0,1]^{t}$ denote the North pole ( $t$ denotes transpose). To each function $f$ on $S^{2}$, let $f$ denote the function on $\operatorname{SO}(3)$ defined by the rule $\tilde{f}(R)=f(R \hat{k})$. We define the spherical triple correlation of $f$ to be $a_{3, \tilde{f}}$ in eq. (3) for the corresponding function $\tilde{f}$ on $\operatorname{SO}(3)$. This triple correlation is invariant under 3-D rotation of $f$. To see that, suppose $h$ is another spherical function that is a 3-D rotated copy of $f$. Then there exists a rotation $R_{0}$ such that $h(x)=f\left(R_{0} x\right)$ for all $x \in S^{2}$. Thus $\check{h}(R)=h(R \hat{k})=f\left(R_{0} R \hat{k}\right)=\tilde{f}\left(R_{0} R\right)$, or equivalently, the two functions are left translates of each other, and consequently $a_{3, \tilde{h}}=a_{3, \tilde{f}}$

Now suppose we observe $r(x)=f(x)+n(x)$ on $S^{2}$, where $f$ is a determinstic function and $n$ is a sample function from a Gaussian, zero-mean, stationary process $N$. As above, it may be shown that

$$
E\left[a_{3, \tilde{r}^{\prime}}(S, T)\right]=a_{3, \tilde{f}^{\prime}}(S, T),
$$

with $\tilde{r}^{\prime}$ and $\tilde{f}^{\prime}$ denoting respectively the extensions to $\mathrm{SO}(3)$ of the mean-subtracted functions $r-\bar{r}$ and $f-\bar{f}$, the mean being evaluated on the sphere [7, pp 21-22].

\section{BISPECTRUM FORMULA}

In this section, we calculate the Fourier representation of the spherical triple correlation. Fourier analysis on the group $\mathrm{SO}(3)$ is determined by the irreducible unitary representations of the group $[6, \mathrm{Ch}$. I]. The latter are matrix valued functions $D_{\ell}, \ell \geq 0$, whose coefficients are called generalized spherical harmonics. For each $\ell$, the function $D_{\ell}$ maps $\mathrm{SO}(3)$ into the group of unitary matrices of dimension $2 \ell+1$, in such a way that $D_{\ell}(R S)=D_{\ell}(R) D_{\ell}(S)$ for all $S, R$. For any $L_{2}$ function $\tilde{f}$ on $\mathrm{SO}(3)$, we have the Fourier series expansion

$$
\tilde{f}(R)=\sum_{\ell=0}^{\infty} \operatorname{tr}\left[\tilde{F}(\ell) D_{\ell}(R)\right],
$$

where $\operatorname{tr}$ denotes matrix trace, and the Fourier coefficient matrices $F$ are obtained from the matrix-valued integral:

$$
\tilde{F}(\ell)=\int_{S O(3)} \tilde{f}(R) D_{\ell}(R)^{\dagger} d R
$$

Here, the dagger $\dagger$ denotes matrix adjoint. The translation property of the Fourier coefficients is important in the sequel: $\tilde{h}(R)=\tilde{f}(S R)$ for all $R$ if and only if $\tilde{H}(\ell)=$ $\dot{F}(\ell) D_{\ell}(S)$ for all $\ell$.

The triple correlation is a function on $S O(3) \times S O(3)$, and therefore requires for its Fourier expansion Kronecker products of the $D_{\ell}$ matrices. The $(k, \ell)$-th Fourier coefficient matrix of $a_{3, \tilde{f}}$, which we denote $A_{3, j}(k, \ell)$, is computed by the integral

$$
\int_{S O(3)} \int_{S O(3)} a_{3, \tilde{f}}(S, T)\left[D_{k}(S)^{\dagger} \otimes D_{\ell}(T)^{\dagger}\right] d S d T,
$$

where $\otimes$ denotes the matrix Kronecker product. By the Clebsh-Gordan formula, the matrix $D_{k}(S) \otimes D_{\ell}(T)$ reduces, when $S=T$, into the following direct sum of smaller matrices:

$$
C_{k \ell}\left[D_{k+\ell}(S) \oplus D_{k+\ell-1}(S) \oplus \cdots \oplus D_{|k-\ell|}(S)\right] C_{k \ell}^{\dagger}
$$

Here $C_{k \ell}$ is the unitary matrix of Clebsh-Gordan coefficients (independent of $S$ ), and $\rightarrow$ denotes matrix direct sum.

The discussion above gives us the machinery required to compute the spherical bispectral coefficients [7, pg 78].

Proposition 1 Let $\tilde{f}$ be any $L_{2}$ function on $S O(3)$ with Fourier coefficients $\tilde{F}(\ell), \ell \geq 0$. For each $k$ and $\ell$, we have

$$
\begin{aligned}
A_{3, \tilde{f}}(k, \ell)= & {[\tilde{F}(k) \otimes \tilde{F}(\ell)] C_{k \ell}\left[\tilde{F}(k+\ell)^{\dagger} \oplus\right.} \\
& \left.\tilde{F}(k+\ell-1)^{\dagger} \cdots \oplus \tilde{F}(|k-\ell|)^{\dagger}\right] C_{k \ell}^{\dagger} .
\end{aligned}
$$

Intuitively, the spherical bispectrum formula follows from the Euclidean formula (2) by taking into accout the Kroneckerproduct decomposition formula (5) on the group $\mathrm{SO}(3)$. (For the group $\mathbb{R}$, the Kronecker-product decomposition of irreducible unitary representations is simply the familiar expression $e^{i \lambda x} e^{i \nu x}=e^{i(\lambda+\nu) x}$.)

If $f$ is any function on the sphere, and $\tilde{f}$ is its extension to SO (3) by the North pole method of $\S I$, then we call $A_{3, \bar{f}}$ the spherical bispectrum of $f$.

\section{BISPECTRUM RECOVERY ALGORITHM}

The formula in Proposition 1 suggests that it is possible to extract the underlying Fourier coefficients recursively from the bispectrum. We demonstrate below an algorithm accomplishing this for functions $\tilde{f}$ defined on SO(3), and later, we show how the technique applies to spherical functions.

Our algorithm makes use of the following facts from matrix theory ([8]). First, any positive definite matrix $H$ has unique "positive square root", i.e., a positive definite matrix $H_{+}^{\frac{1}{2}}$ such that $H_{+}^{\frac{1}{3}} H_{+}^{\frac{1}{3}}=H$. Second, any nonsingular matrix $A$ has a unique polar decomposition $A=H_{+} U$. where $H_{+}=\left(A A^{\dagger}\right)_{+}^{\frac{1}{2}}$, and $U$ is a unitary matrix.

Now let $L>0$, and let $\tilde{f}$ be any real-valued function on $\mathrm{SO}(3)$ whose Fourier coefficients are such that $\tilde{F}(\ell)$ is a nonsingular matrix for each $\ell \leq L$, and furthermore, $\tilde{F}(\ell)=0$ if $\ell>L$. Since $\tilde{f}$ is real-valued, it is easily shown that 
$\operatorname{det}[\check{F}(1)]$ is a real number. Assume, for now, that it is positive. Our algorithm for recovering $\dot{f}$ from its bispectrum $A_{3, j}$ proceeds in three steps.

1. Since $\tilde{f}$ is real-valued, $F(0)$ is a real number, and by Prop. $1, A_{3, j}(0,0)=\tilde{F}(0)^{3}$, and thus $\tilde{F}(0)=$ $\sqrt[3]{A_{3, j}(0,0)}$.

2. We estimate the first Fourier coefficient matrix $\tilde{F}(1)$. Because $\tilde{F}(0)$ is, by assumption, a nonzero real number, we use the formula in Prop. 1 and find that

$$
\frac{A_{3, \tilde{f}}(1,0)}{F(0)}=\left[\dot{F}(1) \tilde{F}(1)^{\dagger}\right] \text {. }
$$

The matrix on the right hand side above is positive definite. Let $\hat{F}(1)=\left(\frac{A_{3, f}(1,0)}{F(0)}\right)_{+}^{\frac{1}{2}}$ be its positive square-root. It can be shown ([7, pp 116-118]) that if $\hat{F}(1)$ is constructed in this way, then there exists $S$ in $\mathrm{SO}(3)$ such that

$$
\hat{F}(1)=\tilde{F}(1) D_{1}(S)
$$

3. If $L=1$, then we are done. Otherwise, the following recursion produces matrices $\hat{F}(2), \ldots, \hat{F}(L)$, such that for all $2 \leq \ell \leq L$ we have that $\hat{F}(\ell)=\tilde{F}(\ell) D_{\ell}(S)$ for the same $S$ as in Step 2 . Since we know $\hat{F}(1)$ and $A_{3 . \hat{f}}(1,1)$, we obtain $\hat{F}(2)$ from the upper-left $5 \times 5$ subinatrix of the following $9 \times 9$ matrix:

$$
C_{11}^{\dagger}\left[\hat{F}(1)^{-1} \otimes \hat{F}^{\prime}(1)^{-1}\right] A_{3, j}(1,1) C_{11} \text {. }
$$

Explanation: all terms above are known, and if we substitute for $\hat{F}(1)$ and $A_{3, \tilde{f}}(1,1)$, and use the reduction formula (5), then we find that

$$
\begin{array}{r}
C_{11}^{\dagger}\left[\hat{F}(1)^{-1} \otimes \hat{F}(1)^{-1}\right] A_{3, j}(1,1) C_{11} \\
{\left[D_{2}(S)^{\dagger} \tilde{F}(2)^{\dagger}\right] \oplus\left[D_{1}(S)^{\dagger} \tilde{F}(1)^{\dagger}\right] \oplus \tilde{F}(0)}
\end{array}
$$

The upper left $5 \times 5$ submatrix of the right hand side is exactly the matrix $\left[\check{F}(2) D_{2}(S)\right]^{\dagger}$, and we set its adjoint equal to $\hat{F}(2)$. Having obtained $\hat{F}(2)$, we estimate $\dot{F}(\ell)$ for any $\ell>2$ from the adjoint of the upper left $(2 \ell+1) \times(2 \ell+1)$ submatrix of the following matrix:

$$
C_{(\ell-1) 1}^{\dagger}\left[\hat{F}(\ell-1)^{-1} \otimes \hat{F}(1)^{-1}\right] A_{3, f}(\ell-1,1) C_{(\ell-1) 1}
$$

The same argument as above shows that $\hat{F}(\ell)=$ $\tilde{F}(\ell) D_{\ell}(S)$. After iterating the recursion until $\ell=L$, the function $\hat{f}$ on $\mathrm{SO}(3)$ obtained by Fourier series expansion with the coefficients $\tilde{F}(0), \hat{F}(1), \ldots, \hat{F}(L)$, is such that $\hat{f}(R)=\tilde{f}(S R)$ for all $R$.

The assumption that $\operatorname{det}[\tilde{F}(1)]>0$ is not critical. Instead of selecting $\hat{F}(1)$ to be the positive definite square root of $\tilde{F}(1) \tilde{F}(1)^{\dagger}$, we may choose $\hat{F}(1)$ to be any square root such that $\operatorname{det}[\hat{F}(1)]=\operatorname{det}[\tilde{F}(1)]$, e.g., by multiplying the top row of the positive definite square root matrix by -1 if necessary. We do not know $\operatorname{det}[F(1)]$ a priori, but if we store it as "side information" along with the triple correlation, then we obtain a complete (left) translation-invariant description for any real-valued bandlimited function on $S O(3)$. Note that $\operatorname{det}[\tilde{F}(1)]$ remains invariant under translation on $S O(3)$, i.e., if $\tilde{f}(R)=\tilde{h}(S R)$, then $\tilde{F}(1)=\tilde{H}(1) D_{1}(S)$, but since $\operatorname{det}\left[D_{1}(S)\right]=+1([9, \mathrm{pg} 47])$ we obtain that $\operatorname{det}[\tilde{F}(1)]=\operatorname{det}[\tilde{H}(1)]$. To sum up, any real-valued bandlimited function $\tilde{f}$ on $S O(3)$, whose coefficient matrices are all nonsingular up to the bandlimit, can be recovered completely-up to a single left translation on $\mathrm{SO}(3)$-if both its triple correlation and the value of $\operatorname{det}[\tilde{F}(1)]$ is known, and the algorithm described above is used.

\section{SPHERICAL FUNCTIONS}

The algorithm described in the previous section is formulated for functions on $\mathrm{SO}(3)$, but we need additional steps to use it for spherical functions. Let $\tilde{f}$ be the extension to $\mathrm{SO}(3)$ of a spherical function $f$ via the North pole mapping. The Fourier coefficient matrices $\tilde{F}(0), \tilde{F}(1), \ldots$ of any function $\tilde{f}$ obtained in this way have at most rank 1 . To prove this, consider that $\tilde{f}$ is invariant under any rotation $Q$ that leaves the North pole fixed, i.e.,

$$
\tilde{f}(R Q)=f(R Q \hat{k})=f(R \hat{k})=\tilde{f}(R) .
$$

The set of all rotations $Q$ that fix the North pole forms a subgroup $\mathcal{Q}$ of $\mathrm{SO}(3)$. Thus we can average each Fourier coefficient matrix $\tilde{F}(\ell)$ over $\mathcal{Q}$ in without changing the value of the coefficient. It is easily shown that this implies $\tilde{F}(\ell)=$ $P_{\ell} \hat{F}(\ell)$, where

$$
P_{\ell}=\int_{\mathcal{Q}} D_{\ell}(Q) d Q .
$$

The matrix $P_{\ell}$ is an orthogonal projection, whose elements are zero everywhere except for the exact center of the matrix, which has the value 1 ; thus the rank of $\tilde{F}(\ell)$ cannot exceed one [7, pg 95].

Although the Fourier coefficients of an extended spherical function $\tilde{f}$ are singular, we may augment them so that they become nonsingular, compute the resulting bispectrum, and then use the recovery algorithm in §IV. This allows us to average multiple observations of a rotating spherical function, a procedure that is described in §VI. We focus now on the method of constructing nonsingular Fourier matrices.

Since $\tilde{F}(\ell)=P_{\ell} \tilde{F}(\ell)$, the entries of the $2 \ell+1$-dimensional matrix $\tilde{F}(\ell)$ are zero except for the middle row. Assume that at least one of the middle row elements is nonzero; then we may substitute for the $2 \ell$ remaining rows linearly independent vectors so that the resulting matrix, denoted $\dot{F}(\ell)$, is nonsingular. (We are finding a nonsingular matrix $\check{F}(\ell)$ such that $\tilde{F}(\ell)=P_{\ell} \check{F}(\ell)$.) Moreover, we may choose the additional vectors so that the function $\check{f}$ defined by the coefficients $\check{F}(0), \check{F}(1), \ldots, \check{F}(L)$ is real-valued.

Now the function $f$ is uniquely determined by its bispectrum $A_{3, j}$ up to a 3-D rotation, i..e, from $A_{3, j}$ we can recover Fourier coefficients $\tilde{F}$ such that for some $S$ and all $0 \leq \ell \leq L$, we have $\tilde{F}(\ell)=\tilde{F}(\ell) D_{\ell}(S)$. We then obtain the 
original Fourier coefficients (up to a 3-D rotation) by composition : $P_{\ell} \tilde{F}(\ell)=\tilde{F}(\ell) D_{\ell}(S)$. Thus the bispectrum of $\check{f}$ uniquely determines $\tilde{f}$, and consequently also the original spherical function $f$, up to a 3-D rotation.

\section{APPLICATIONS}

We outline two applications of the spherical bispectrum, one to matching spherical functions, and the other to averaging multiple views of a rotating spherical function.

Since the spherical bispectrum is invariant under 3-D rotation of the underlying function, we check whether two spherical functions $p$ and $q$ are simply 3-D rotations of each other by comparing their bispectra. The bispectrum test has two appealing properties. First, the recovery algorithm in $\$ 4$, although formulated for functions on $\mathrm{SO}(3)$ with nonsingular coefficients, shows that bispectral coefficients are potentially powerful enough to uniquely determine the underlying function and its translates. Second, the insensitivity of bispectral coefficients to additive Gaussian noise suggests that any test involving matching bispectral coefficients should be insensitive to measurement noise. In practice, however, the bispectra of rotated copies of the same function will not match exactly, and thus we need to check if their difference is less than some threshold, where the latter is determined by measuring or calculating the probability distribution of the bispectral coefficients. We leave this topic for future investigation.

Suppose now that we have several noisy observations $f_{1}$. $\ldots, f_{n}$, of a single spherical function $f$, where the function may be rotating by an unknown amount in between successive observations, and the noise is independent in each observation. Since the signal is rotating, simply averaging the observations would average out the signal. Instead, we may use the following bispectral technique to average the noise without averaging out the signal. For each observation, we compute the spherical bispectrum, and average the resulting bispectra over all the observations. In doing this, we are averaging noise without averaging out information about the signal. Unfortunately, we cannot apply the algorithm of $\$ 4$ to recover the original signal from the averaged spherical bispectrum $\left\langle A_{3, j}\right\rangle$, because the underlying Fourier coefficient matrices are singular. However, we may recover the underlying signal if we select any one of the observations, say $f_{n}$, form the augmented function $\dot{f}_{n}$ on $\mathrm{SO}(3)$ with nonsingular coefficients, and compute the bispectrum $A_{3, f_{n}}$. The nonzero coefficients of $\left\langle A_{3, j}\right\rangle$ form a proper subset of the coefficients of $A_{3, f_{n}}$, and thus we may improve signal-to-noise ratio in the reconstruction by substituting in the appropriate locations of each matrix $A_{3},(k, \ell)$ the coefficients of the averaged matrix $\left\langle A_{3, j}(k, \ell)\right\rangle$. Numerical results obtained by using this procedure will be reported in future work.

\section{SUMMARY}

In this paper, we formulate the appropriate bispectrum for spherical functions, and show that it in unbiased in Gaussian noise and invariant under 3-D rotation of the underlying function. We demonstrate an algorithm for recover- ing functions on the 3-D rotation group from their bispectra, and we describe how that algorithm may be used with spherical functions by augmenting the Fourier coefficient matrices. Finally, we describe applications of the spherical bispectrum to the problem of checking whether two spherical functions are simply 3-D rotations of each other, and to the problem of averaging multiple views of a single rotating spherical function.

\section{REFERENCES}

[1] R. A. Nash and S. K. Jordan, "Statistical geodesy: an engineering perspective," Proceedings of the IEEE, Vol. 66, No. 5, pp 532-550, May 1978.

[2] C. W. Chen and T. S. Huang, "Left ventricle motion an alysis by hierarchical decomposition," in Proceedings ICASSP-92, Vol. 3, pp 273-276, 1992.

[3] M. D'Zmura, "Shading ambiguity: Reflectance and illumination," in Computational models of visual processing, M. S. Landy and A. J. Movshon, Eds., Cambridge: MIT Press, pp 187-207,1991.

[4] A. W. Lohmann and B. Wirnitzer, "Triple correlations," Proceedings of the IEEE, Vol. 72, pp 889-901, 1984.

[5] J. I. Yellott Jr., and G. J. Iverson, "Uniqueness results for generalized autocorrelations," Journal of the Optical Society of America A, Vol. 9, pp 388-404, 1992.

[6] I. M. Gel'fand, R. A. Minlos, and Z. Ya. Shapiro, Representations of the rotation and Lorentz groups and their applications, Oxford: Pergammon Press, 1963.

[7] R. Kakarala, Triple correlation on groups, Ph.D. Thesis, Department of Mathematics, University of California, Irvine, 1992.

[8] P. Lancaster and M. Tismenetsky, The theory of matrices, Orland: Academic Press, 1985.

[9] L. C. Biedenharn and J. D. Louck, Angular momentum in quantum physics: Theory and application, Reading, MA: Addison-Wesley, 1981. 